\title{
APH-1a Is the Principal Mammalian APH-1 Isoform Present in $\gamma$-Secretase Complexes during Embryonic Development
}

\author{
Guojun Ma, ${ }^{1}$ Tong Li, ${ }^{2}$ Donald L. Price,,${ }^{1,2,3}$ and Philip C. Wong ${ }^{1,2}$ \\ Departments of ${ }^{1}$ Neuroscience, ${ }^{2}$ Pathology, and ${ }^{3}$ Neurology, Johns Hopkins University School of Medicine, Baltimore, Maryland 21205
}

\begin{abstract}
APH-1 (anterior pharynx defective) along with nicastrin and PEN-2 (presenilin enhancer) are essential components of the presenilin (PS)-dependent $\gamma$-secretase complex. There exist three murine Aph-1 alleles termed Aph-1a,Aph-1b, and Aph-1cthat encode four distinct APH-1 isoforms: APH-1aL and APH-1aS derived from differential splicing of Aph-1a, APH-1b, and APH-1c. To determine the contributions of mammalian APH-1 homologs in formation of functional $\gamma$-secretase complexes, we generated $A p h-1 a^{-/-}$mice and derived immortalized fibroblasts from these embryos. Compared with littermate controls, the development of $A p h-1 a^{-1-}$ embryos was dramatically retarded by embryonic day 9.5 and exhibited patterning defects that resemble, but are not identical to, those of Notch1, nicastrin, or PS null embryos. Moreover, in immortalized $A p h-1 a^{-1-}$ fibroblasts, the levels of nicastrin, PS fragments, and PEN-2 were dramatically decreased. Consequently, deletion of $A p h$ - $1 a$ resulted in significant reduction in levels of high-molecular-weight $\gamma$-secretase complex and secretion of $\beta$-amyloid $(\mathrm{A} \beta)$. Importantly, complementation analysis revealed that all mammalian APH-1 isoforms were capable of restoring the levels of nicastrin, PS, and PEN-2, as well as A $\beta$ secretion in Aph-1 $a^{-1-}$ cells. Together, our findings establish that APH-1a is the major mammalian APH-1 homolog present in PS-dependent $\gamma$-secretase complexes during embryogenesis and support the view that mammalian APH-1 isoforms define a set of distinct functional $\gamma$-secretase complexes.
\end{abstract}

Key words: Aph-1a null mice; APH-1 isoforms; $\gamma$-secretase complexes; nicastrin; presenilins; Alzheimer’s disease

\section{Introduction}

Alzheimer's disease (AD), the most common cause of dementia in the elderly, is characterized by the deposition of $\beta$-amyloid $(\mathrm{A} \beta)$ and the presence of neurofibrillary tangles in the brain (Price and Sisodia, 1998; Petersen, 2003). A $\beta$ peptides are generated by sequential endoproteolytic cleavages of $\beta$-amyloid precursor protein (APP) by BACE1 ( $\beta$-site APP-cleaving enzyme) and $\gamma$-secretase complex (Wong et al., 2002). The presenilins (PSs), which when mutated cause autosomal dominant AD (Sisodia and St. George-Hyslop, 2002), are critical for the regulated intramembraneous proteolysis of a growing number of type I transmembrane proteins, including APP and Notch (Selkoe and Kopan, 2003; Wolfe and Kopan, 2004). Recent studies (Edbauer et al., 2003; Farmery et al., 2003; Kim et al., 2003; Kimberly et al., 2003; Takasugi et al., 2003) support the view that PSs form highmolecular-weight complexes with several other transmembrane proteins critical for $\gamma$-secretase activity, including nicastrin (NCT) (Yu et al., 2000; Li et al., 2003), APH-1 (anterior pharynx defective) (Francis et al., 2002; Goutte et al., 2002; Lee et al., 2004), and PEN-2 (presenilin enhancer) (Francis et al., 2002).

One essential member of $\gamma$-secretase complex is APH-1, a sevenpass membrane protein (Francis et al., 2002; Fortna et al., 2004) that

Received Sept. 14, 2004; revised Nov. 12, 2004; accepted Nov. 14, 2004.

This work was supported by National Institutes of Health Grants R01 NS41438 and R01 NS45150, the American Health Assistance Foundation, the Adler Foundation, the Rotary "Coins for Alzheimer's Research Trust" Fund, and the Bristol-Myers Squibb Foundation. We thank G. Thinakaran for PEN-2 antisera and V. Nehus for technical support.

Correspondence should be addressed to Dr. Philip C. Wong, Department of Pathology, Johns Hopkins University School of Medicine, 720 Rutland Avenue, Ross 558, Baltimore, MD 21205. E-mail: wong@jhmi.edu.

DOI:10.1523/JNEUROSCI.3814-04.2005

Copyright $\odot 2005$ Society for Neuroscience $\quad$ 0270-6474/05/250192-07\$15.00/0 forms a stable subcomplex with nicastrin (Gu et al., 2003; Hu and Fortini, 2003; LaVoie et al., 2003; Takasugi et al., 2003). Unlike Caenorhabditis elegans and Drosophila in which a single Aph-1 gene exits, three Aph-1 genes are found in mice; Aph-1a is localized to chromosome 3, whereas Aph-1b and Aph-1c are arranged in tandem $(\sim 20$ $\mathrm{kb}$ apart) on chromosome 9. Because two isoforms of APH-1a can be generated from alternative splicing of Aph-1a, APH-1a long form (APH-1aL) and APH-1a short form (APH-1aS), a total of four distinct APH-1 isoforms can be found in mice. Although APH-1a has $\sim 55 \%$ similarity with APH-1b/APH-1c, whereas APH-1b and APH-1c share 95\% similarity, it remains undefined whether different mammalian APH-1 isoforms possess similar or distinct roles in $\gamma$-secretase activity or as to their relative contributions to $\gamma$-secretase complexes during development, maturation, and aging.

To begin to assess the physiological roles of mammalian $\mathrm{APH}-1$ isoforms, their contributions to formation of functional $\gamma$-secretase complexes, and the mechanism whereby APH-1 facilitates the complex assembly, we generated and analyzed Aph$1 a$-deficient mice and immortalized fibroblasts derived from

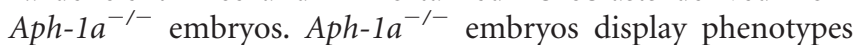
that resemble, but are not identical to, those of Notch1 (Swiatek et al., 1994; Conlon et al., 1995; Huppert et al., 2000), nicastrin (Li et al., 2003), or PS (Donoviel et al., 1999; Herreman et al., 1999) null embryos. Importantly, the deletion of $A p h-1 a$ led to a significant reduction in nicastrin, PS, and PEN-2, accompanied by decrease in high-molecular-weight $\gamma$-secretase complex and secretion of A $\beta$ peptides. Moreover, complementation analysis using Aph$1 a^{-/-}$cells showed that all murine APH-1 isoforms are functional in $\gamma$-secretase activity. Thus, our results establish that APH-1a is the principal APH-1 isoform present in $\gamma$-secretase 
complexes during mouse embryogenesis and support the view that mammalian APH-1 isoforms define a set of distinct functional $\gamma$-secretase complexes.

\section{Materials and Methods}

Cloning and transfection. Aph-1aS and Aph-1bcDNAs were generated by reverse transcriptase-PCR of mRNA extracted from tail of mice using the following primers: 5'-CAGCTCCCTTTCGCCTTCCTTG, 5'-GTCCTTACACGAAAGGCTGCGC, 5'-ATGACGCTGCCTGTGTTCTTCG, and 5'-TCTGGAGCGT TGGTTGTAAAGAAGAAAATC. Aph-1aL EST (expressed sequence tagged) clone was purchased from Invitrogen [EST library (San Diego, CA)]. All Aph-1 cDNAs were then subcloned into pcDNA3.1-V5/His vector using a Topo expression kit (Invitrogen). Aph$1 a^{-/-}$cells were transfected with expression vectors encoding APH-1aL, APH-1aS-V5, APH-1b-V5, or APH-1c using the Lipfectamine 2000 reagent (Invitrogen). Cells were collected $48 \mathrm{hr}$ after transfection and analyzed by protein blotting.

Generation of Aph-1a ${ }^{-1-}$ mice. The Aph-1a gene, isolated from a murine genomic $129 / \mathrm{SvJ}$ library (Stratagene, La Jolla, CA) using a murine Aph-1a cDNA probe (reverse transcriptase-PCR extracted from tail of mice using the primers 5'-GTTATCATCCTG GTGGCGGGAG and 5' GCTGAAGTCAGGAAGTAATA GGGTGAGTC) was characterized by a series of restriction enzyme and DNA sequencing analysis. Aph-1a genomic sequence was obtained from the National Center for Biotechnology Information mouse genome database. In the Aph-1a targeting vector, a $2.5 \mathrm{~kb}$ fragment containing exon 1 to intron 5 and part of exon 6 of the Aph-1a gene was replaced with a neomycin-resistant (Neo) gene. The linearized Aph-1a targeting vector was electroporated into 129/SvJ embryonic stem (ES) cells, and targeted clones were screened by Southern blot analysis using a flanking probe generated by PCR of mouse DNA via primers 5'-GAATTCACTGCTTTCTCCCCGC and 5-CCTCTCGGAAGTGTACCCCAG.

Eight independent targeted clones were injected into C57BL/6 mouse blastocysts to generate Aph-1a chimeric mice. Mating of chimeric mice to C57BL/6 mice produced offspring bearing one inactivated Aph-1a allele

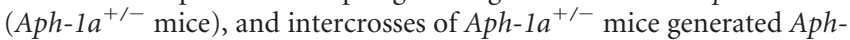
$1 a^{-1-}$ mice. DNA extracted from tail clips or yolk sacs of mice were genotyped by PCR using the following sets of primers: for the endogenous allele, 5' -GCTGTCTCTGTCCTTCTACAGGAG and 5' - CGGAAGATCACCCATCTCCATCC; and for the targeted allele, 5' - GTACACCATCACCCGACTGTC and 5' -CTACCCGCTTCCATTGCTCAG.

Characterization of the Aph-1a ${ }^{-1-}$ embryos. Embryos were dissected and examined by light microscopy or fixed in $4 \%$ paraformaldehyde, embedded in paraffin, sectioned, and stained with hematoxylin and eosin (H\&E), or processed for whole-mount in situ hybridization using Dll1 (Delta-like 1) as probe generated using a Digoxigenin RNA labeling kit (Roche, Indianapolis, IN) following previously established protocols.

Generation of immortalized Aph-1a ${ }^{-1-}$ fibroblasts. Primary fibroblasts were cultured from Aph-1 $a^{+/+}, A p h-1 a^{+/-}$, and Aph-1 $1 a^{-/-}$embryos at embryonic day 9 (E9) to E9.5. Briefly, the embryos were minced, suspended in $0.25 \%$ trypsin, and incubated at $37^{\circ} \mathrm{C}$ for $15 \mathrm{~min}$. The tissues were then transferred to DMEM supplemented with $10 \%$ fetal bovine serum and dissociated by repeated trituration. The dispersed cells were plated in one well of a 48-well plate and subsequently transfected with large $\mathrm{T}$ antigen (Cai et al., 2001). Immortalized cells were then passaged and frozen in liquid nitrogen for later use.

Antibodies and protein blotting. Protein extracts were resolved on $4-20 \%$ Tris-glycine or $10-20 \%$ tricine gels, transferred to polyvinylidene difluoride membranes, and probed with the following antibodies: antihuman APH-1aL and anti-human APH-1b/c (1:1000; Covance, Princeton, NJ); anti-nicastrin (NCT-3925; 1:2000) (Li et al., 2003); anti-PS1C-terminal fragment (CTF) (1:2500), anti-PS1-N-terminal fragment (NTF) (1:2000), and anti-PS2-CTF (1:2000) (Thinakaran et al., 1996); anti-PEN-2 (1:2000) (Vetrivel et al., 2004); anti-APP-CTF (CT15, 1:2000) (Chemicon, Temecula, CA); anti-V-5-HRP (1:2000) (Invitrogen); rabbit anti-superoxide dismutase (SOD1) antibody (1:5000; previously generated in our laboratory); and mouse monoclonal antibody against actin (Chemicon). Protein extracts were analyzed by immunoblotting with enhanced chemiluminescence.
Blue native-PAGE analysis. To prepare the membrane fraction, cells were washed with PBS, resuspended in 20 mM HEPES, pH 7.2, with 150 $\mathrm{mm} \mathrm{NaCl}, 2 \mathrm{~mm}$ dithiothreitol, 2 mM EDTA, 10\% glycerol, and a mixture of protease inhibitors (Roche), and homogenized with 30 strokes in a glass Dounce homogenizer. Cell debris and nuclei were removed by centrifugation at $800 \times g$ for $10 \mathrm{~min}$. The supernatants were centrifuged at $100,000 \times g$ for $60 \mathrm{~min}$ to collect the membrane fractions. Membrane preparations were resuspended in $20 \mathrm{~mm}$ Tris $\mathrm{HCl}, \mathrm{pH} 7.4$, with $0.5 \%$ $N$-dodecyl $\beta$-D-maltoside, $500 \mathrm{~mm}$ e-amino caproic acid, 2 mм EDTA, and $10 \%$ glycerol, incubated on ice for $60 \mathrm{~min}$, and then centrifuged at $100,000 \times g$ for $60 \mathrm{~min}$. The membrane protein extracts were subjected to blue native-PAGE essentially as described previously (Schagger and von Jagow, 1991). Marker proteins were BSA (66 kDa and dimer at 132 $\mathrm{kDa}), \beta$-amylase $(200 \mathrm{kDa})$, apoferritin $(443 \mathrm{kDa})$, and thyroglobulin $(667 \mathrm{kDa})$ (Sigma, St. Louis, MO). After electrophoresis, protein complexes were analyzed by immunoblotting with antisera specific to nicastrin and PS1.

$A \beta$ assays. APP recombinant adenovirus expressing human APP Swedish mutation (APPSwe) was constructed as described previously (Cai et al., 2001). To examine APP processing, immortalized fibroblasts were infected with $5 \times 10^{6}$ plaque-forming units of adenovirus for $24-36 \mathrm{hr}$. $A \beta 40$ and $A \beta 42$ levels from culture supernatants of cells were measured using a quantitative sandwich ELISA kit (Biosource International, Camarillo, CA) that specifically detects human $\mathrm{A} \beta 40$ or $\mathrm{A} \beta 42$.

\section{Results}

\section{Aph-1a-deficient mice are embryonic lethal}

To determine the physiological role of Aph-1a and to assess whether different mammalian isoforms of APH-1 confer functional differences in $\gamma$-secretase activity, we examined functional consequences of ablating Aph-1a in mice by using a homologous recombination strategy in ES cells to inactivate this gene.

To generate the Aph-1a targeting vector, a $2.5 \mathrm{~kb}$ fragment containing exon 1 to intron 5 and part of exon 6 was replaced with a neomycin-resistance gene (Fig. 1 A). 129/SvJ ES cells were transfected with the linearized $A p h-1 a$ targeting vector, and 19 clones (of 220 screened) were targeted at the Aph-1a locus (Fig. 1B). Eight ES cell clones with a targeted $A p h-1 a$ allele $\left(A p h-1 a^{+/-}\right)$ were injected into C57BL/6 blastocysts to generate Aph-1a chimeric mice. Mating of chimeric mice to C57BL/ 6 mice produced Aph-1 $1 a^{+/-}$mice. However, genotypic analysis of postnatal progeny from intercrosses of $A p h-1 a^{+/-}$mice revealed the presence of

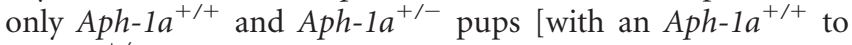
$A p h-1 a^{+/-}$mice ratio of 0.5 (Table 1)], suggesting that Aph-1a knock-out mice might be embryonic lethal.

To determine the age and stage at which embryos died, we collected embryos from E8 to E16. Whereas Aph-1 $1 a^{+/+}$and Aph$1 a^{+/-}$embryos were identified during these time points (Fig.

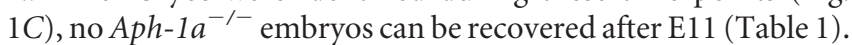
RNA analysis from yolk sacs of $A p h-1 a^{+/+}, A p h-1 a^{+/-}$, and Aph$1 a^{-1-}$ embryos confirmed the absence of Aph-1a mRNA from Aph-1a $a^{-1-}$ embryos (Fig. 1D).

\section{APH-1a is the major mammalian APH-1 isoform required for Notch signaling during embryogenesis}

To determine whether the Aph-1a null phenotype resembles that of Notch1 (Swiatek et al., 1994; Conlon et al., 1995; Huppert et al., 2000), nicastrin (Li et al., 2003), or PS (Donoviel et al., 1999; Herreman et al., 1999) null embryos, we undertook a series of morphological studies to characterize the embryonic phenotypes

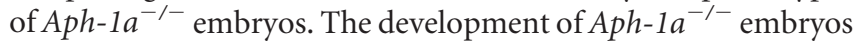
was dramatically retarded by E9.5 when compared with Aph$1 a^{+/+}$and Aph-1 $a^{+/-}$littermate controls (Fig. 2A,B), and growth is arrested by E10.5 (Fig. 2C). Although some E11 Aph$1 a^{-1-}$ embryos can be recovered, no heartbeats can be detected 


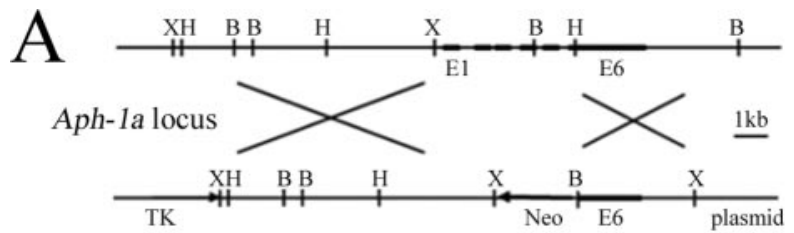

Targeting vector
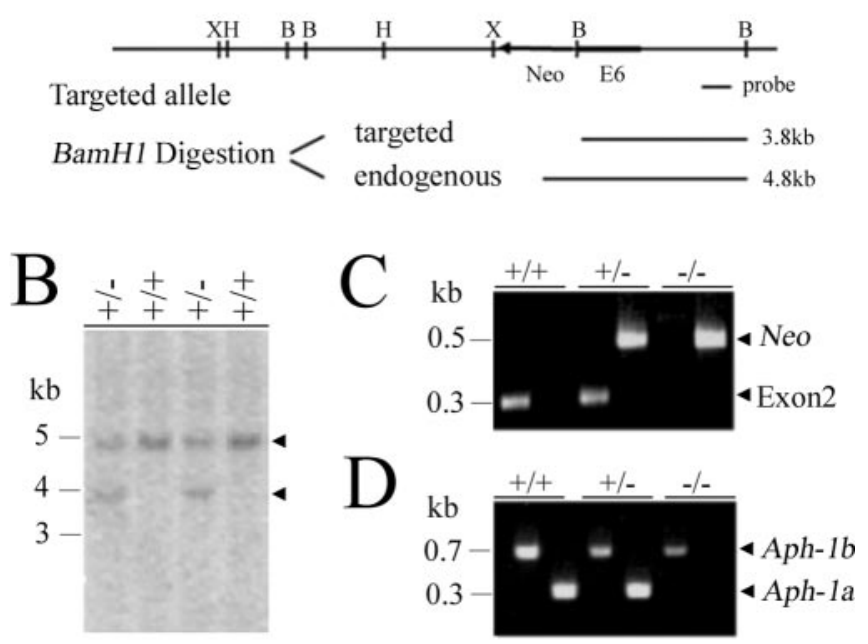

Figure 1. Targeted disruption of the Aph-1a gene by homologous recombination. A, Maps of the Aph-1a locus, the targeting vector, and the disrupted $A p h$ - $1 a$ allele. Black boxes indicate exons 1 through 6. Neo, Neomycin gene; TK, HSV thymidine kinase gene; X, Xbal; H, Hindlll; B, $B a m H I$. Lines below the maps denote expected sizes for BamHI-digested fragments detected by a 3 '-flanking probe (black bar) for targeted and endogenous Aph-1a alleles. B, Analysis of genomic DNA from ES cells by Southern blot. The BamHl fragments detected for endogenous (top arrowhead, $4.8 \mathrm{~kb}$ ) and targeted (bottom arrowhead, $3.8 \mathrm{~kb}$ ) Aph-1a alleles with the 3' probe are indicated. C, $P C R$ analysis of yolk sac DNA from embryos at $E 9.5$ by $P C R$ using specific sets of primers to detect endogenous (Exon2) and targeted (Neo) Aph-1a alleles. D, Total RNA

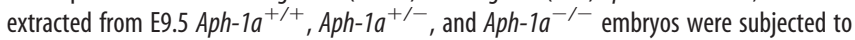
reverse transcriptase-PCR analysis using primers specific for either Aph-1a or Aph-1b RNA.

Table 1. Progenies of crosses of $A p h-1 a^{+/-}$mice

\begin{tabular}{|c|c|c|c|c|c|}
\hline Age & $\begin{array}{l}\text { Number } \\
\text { of litters }\end{array}$ & $\begin{array}{l}\text { Total number } \\
\text { of pups }\end{array}$ & Aph-1a $a^{+/+}$ & Aph-1a $a^{+/-}$ & Aph-1 $1 a^{-/-}$ \\
\hline E8-E8.5 & 3 & 21 & 4 & 11 & 6 \\
\hline E8.5-E9 & 7 & 78 & 22 & 40 & 16 \\
\hline E9-E9.5 & 6 & 56 & 15 & 29 & 12 \\
\hline E9.5-E10.5 & 10 & 75 & 18 & 40 & 17 \\
\hline E10.5-E11 & 4 & 31 & 8 & 14 & 9 \\
\hline E11-E12 & 3 & 24 & 9 & 15 & 0 \\
\hline E12-E16 & 5 & 34 & 10 & 24 & 0 \\
\hline Adult & 6 & 35 & 11 & 24 & 0 \\
\hline
\end{tabular}

Number of embryos is shown for each genotype at various time points indicated. No $\mathrm{Aph}-1 a^{-/-}$embryos were recovered after E11.

from these embryos. Similar to Notch1 null embryos, defects in vascular morphogenesis of the yolk sac (Fig. 2A), distention of the pericardial sac (Fig. $2 B, C$ ), underdeveloped first branchial arch and heart chambers (Fig. $2 D, E$ ), and kinks in the caudal part

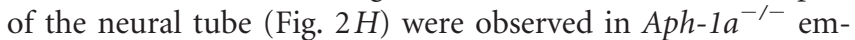
bryos. However, in contrast to Notch1, nicastrin, or PS null em-

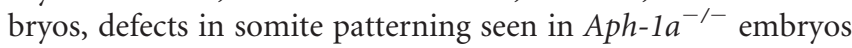
(Fig. $2 F, G$ ) were less severe, particularly the rostral somites, consistent with the finding that $A p h-1 a^{-/-}$embryos survive $1 \mathrm{~d}$ longer than Notch1, nicastrin, or PS null embryos. These results indicate that the presence of APH-1b and APH-1c isoforms accounts for the differences in the phenotype observed between Notch1 and Aph-1a null embryos.
Because Dll1, a ligand of Notch1, is misregulated in the neural tube of PS null embryos (Donoviel et al., 1999), we predict that

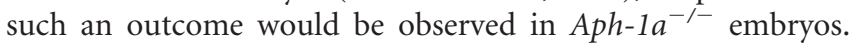
Whole-mount in situ hybridization analysis showed that the level of Dll1 mRNA is indeed upregulated in neural tubes of E8.5 Aph$1 a^{-/-}$embryos (Fig. 2I-K). Because the abnormalities observed in Aph-1 $1 a^{-1-}$ embryos resemble those of Notch1, nicastrin, or PS null embryos and together with observations that $A p h-1 b$ null mice (our unpublished data) and $A p h-1 c$ null mice (B. De Stropper, personal communication) are viable and show no apparent overt phenotype, our results establish that APH-1a is the principle APH-1 isoform required for Notch signaling during mammalian embryogenesis.

\section{APH-1a is the major APH-1 isoform required for the assembly of the $\boldsymbol{\gamma}$-secretase complexes and APP processing} To assess the role of APH-1a in $\gamma$-secretase complex formation and APP processing, we established primary and immortalized fibroblasts from E9.5 control, Aph-1 $\mathrm{a}^{+/-}$, and $\mathrm{Aph}-1 \mathrm{a}^{-/-} \mathrm{em}-$ bryos. We initially confirmed that APH-1aL was absent in Aph$1 a^{-/-}$fibroblasts, whereas in Aph-1 $a^{+/-}$cells, APH-1aL accumulated to $\sim 50 \%$ of the level found in Aph-1 $a^{+/+}$cells (Fig. $3 A$ ). To determine the contribution of APH-1a in $\gamma$-secretase complex formation, we examined levels of nicastrin, PS, and PEN-2 in E9.5 Aph- $1 a^{-/-}$immortalized fibroblasts by protein blot analysis. We found that levels of mature nicastrin, PS1-CTF, PS2-CTF, and PEN-2 were significantly reduced in Aph-1 $a^{-/-}$cells compared with controls (Fig. $3 A$ ). Moreover, formation of highmolecular-weight $\gamma$-secretase complexes was dramatically decreased in Aph-1a $a^{-/-}$fibroblasts (Fig. 3B), as judged by immunoblotting of blue native gel using the nicastrin-specific or PS1-specific antibodies. Importantly, the formation of APH-1/ nicastrin subcomplex was also significantly reduced in Aph-1a null cells (Fig. $3 B$ ). Together with Aph-1 RNAi studies (Lee et al., 2002; Shirotani et al., 2004), our results strongly suggest that APH-1a is required to form a stable subcomplex with nicastrin to facilitate the assembly of PS and PEN-2, leading to the formation of mature $\gamma$-secretase complex.

To confirm that the reduction in level of high-molecularweight $\gamma$-secretase complex observed in $A p h-1 a^{-1-}$ fibroblasts directly impacts on APP processing and leads to a decrease in secretion of $\mathrm{A} \beta$ peptides, we infected $A p h-1 a^{+/+}, A p h-1 a^{+/-}$, and Aph-1 $1 a^{-/-}$fibroblasts with recombinant adenovirus expressing a humanized APP cDNA bearing the Swedish variant (APPSwe) (Cai et al., 2001). Protein blot analysis using CT15, an antibody specific for the $\mathrm{C}$ terminus of APP, revealed an accumulation of APP C-terminal fragments relative to full-length APP in Aph-1a null cells compared with control fibroblasts (Fig. 4A). Furthermore, quantitative sandwich ELISA analysis of conditioned media from Aph-1 $a^{-/-}$cultures expressing APPSwe showed, respectively, $80.4 \pm 8.3$ and $78.8 \pm 7.4 \%$ reduction in the levels of $A \beta 40$ and $\mathrm{A} \beta 42$ peptides (Fig. $4 \mathrm{~B}$ ). Together, these results establish that APH-1a is the principal mammalian APH-1 isoform required for assembly of $\gamma$-secretase complex during embryonic development.

\section{All APH-1 isoforms complement reduced levels of components of $\gamma$-secretase complexes and of secretion of A $\beta$ in Aph-1 $a^{-/-}$cells}

Because there are three mouse Aph-1 alleles that encode four distinct APH-1 isoforms, it is not clear whether APH-1 isoforms possess similar or distinct functions. To examine the functional differences among these variants, we initially performed complemen- 

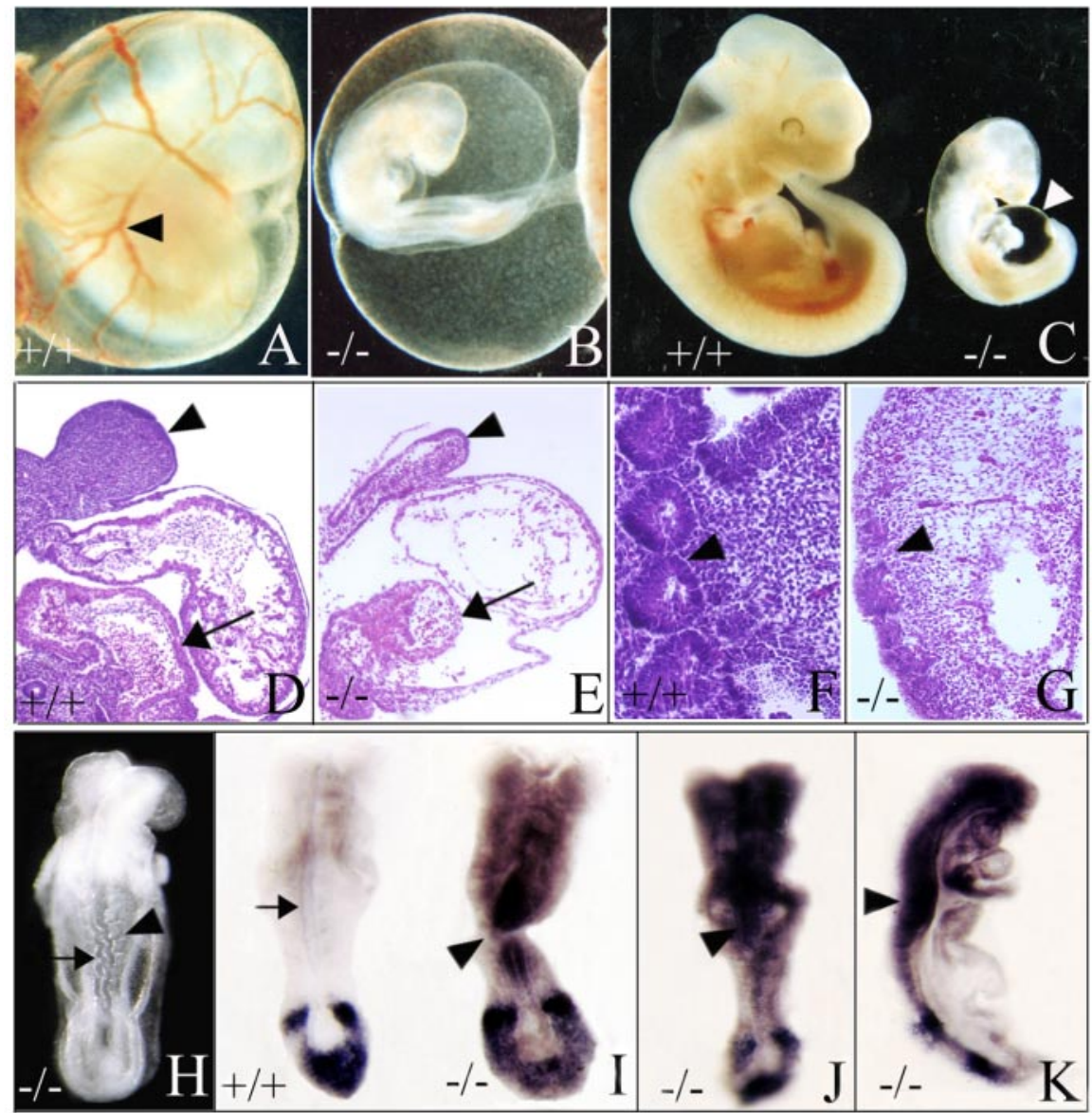

Figure 2. Aph-1a $a^{-1-}$ embryos exhibit a Notch1-like phenotype. $A, B$, Yolk sac vasculature is abnormal in $A p h-1 a^{-1-}$ em-

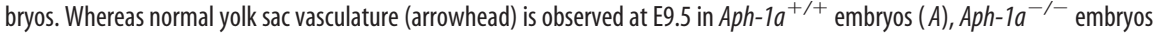
exhibit abnormal vascular morphogenesis of the yolksac $(B)$. C, The development of $A p h-1 a^{-/-}$embryos is dramatically retarded by E10.5 compared with littermate controls. White arrowhead points to distention of pericardial sac in $A p h-1 a^{-1-}$ embryos. D-G, Sagittal sections of Aph-1 $a^{+/+}(D, F)$ and $A p h-1 a^{-/-}(E, G)$ embryos stained with H\&E. Compared with littermate controls $(D)$, Aph-1 $1 a^{-/-}$embryos (E) exhibit patterning defects in the first branchial arch (arrowheads) and the heart chamber (arrows). In contrast to normal somite segmentation observed in $A p h-1 a^{+/+}$embryos ( $F$ ), defective patterning of somites (arrowheads) is seen in $A p h-1 a^{-/-}$

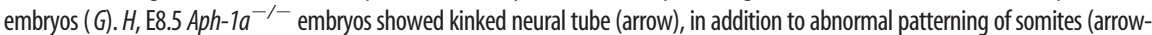
head). $I-K$, Whole-mount in situ hybridization of DII1. Whereas DII1 expression is not detected in neural tubes of Aph-1 ${ }^{+/+}$embryos (arrow in /), increased expression of DII1 is observed in Aph-1a $a^{-/-}$embryos (arrowheads in I-K).

tation experiments by expressing different APH-1 isoforms in Aph-1a null fibroblasts and determined the accumulation of NCT, PS1, and PEN-2. Compared with Aph-1 $1 a^{-/-}$fibroblasts, the levels of mature NCT, PS1-CTF, and PEN-2 were all significantly elevated in Aph-1 $1 a^{-/-}$cells expressing, respectively, APH-1aL, APH-1a5-V5, APH-1b-V5, or APH-1c (Fig. 5A). Moreover, the levels of secreted A $\beta 40$ from Aph-1 $1 a^{-/-}$fibroblasts expressing any of the four APH-1 isoforms were also significantly increased as analyzed by quantitative sandwich ELISA (Fig. 5B). These results demonstrated that APH-1 isoforms are functional and facilitate formation of $\gamma$-secretase complex and support the view that APH-1 isoforms define a set of distinct functional $\gamma$-secretase complexes.

\section{Discussion}

An emerging view is that PSs form high-molecular-weight complexes with three transmembrane proteins (namely, nicastrin, APH-1, and PEN-2) that are critical for generation of functional $\gamma$-secretase complexes (De Strooper, 2003). However, the exact roles of these proteins, particularly for APH-1 in mammals in which three homologous genes exist, in regulation of $\gamma$-secretase complex assembly remain incompletely defined. Despite the demonstration that APH-1 and PEN-2 play important roles in PS-mediated Notch signaling and APP processing (Francis et al., 2002; Goutte et al., 2002) and that PS, nicastrin, APH-1, and PEN-2 comprise the core $\gamma$-secretase complex (Edbauer et al., 2003; Farmery et al., 2003; Kim et al., 2003; Kimberly et al., 2003; Takasugi et al., 2003), the exact mechanism whereby the four components are assembled into the final active complex is ill defined. Particularly intriguing is what physiological roles the three murine Aph-1 genes that encode four APH-1 homologs have. To begin to address this issue, we took a genetic approach to ablate the murine Aph-1 genes. Our demonstration that the phenotype of Aph-1a null embryos resembles that of Notch1 null (Swiatek et al., 1994; Conlon et al., 1995; Huppert et al., 2000), PS null (Donoviel et al., 1999; Herreman et al., 1999), or nicastrin null (Li et al., 2003) embryos not only confirms that APH- 1 a is required for $\gamma$-secretase mediated Notch signaling during mammalian development but establishes that APH-1a is the principal mammalian APH-1 homolog in presenilin-dependent $\gamma$-secretase complexes required for embryonic development. The fact that APH-1b or APH-1c was not able to compensate for the lack of APH-1a in Aph$1 a^{-/-}$mice indicates that the expression levels of $A p h-1 b$ and $A p h-1 c$ genes might be very low or that they are differentially expressed in different cell types during early embryogenesis. Consistent with the idea that APH$\mathrm{lb} / \mathrm{c}$ is expressed to low levels during embryogenesis is the finding that the Aph-1a mRNA is expressed at much higher levels than those of $A p h-1 b$ and $A p h-1 c$ (see the University of California, Santa Cruz mouse gene sorter available at www. genome.ucsc.edu/cgi-bin/hgNear and microarray data). Because the phenotype of $A p h-1 a^{-/-}$mice is not identical to Notch1 null embryos, the low level of APH-1b and APH-1c would contribute to $\gamma$-secretase activity during embryonic de-

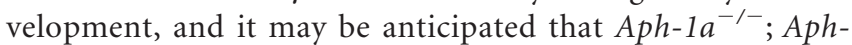

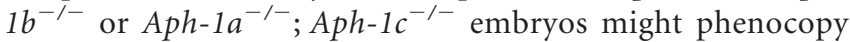
onto the Notch1 null embryos. Indeed, analysis of $A p h-1 a^{-/-}$; Aph-1 $1 b^{-1-}$ embryos revealed phenotypes that are nearly identical to Notch1-deficient embryos (T. Li, G. Ma, H. Wen, F. Davenport, D. L. Price, and P. C. Wong, unpublished observations). Together with the observation that Aph-1a mRNA are reduced during postnatal development (see the University of California, Santa Cruz genome website available at www.genome.ucsc.edu), our results raise the interesting possibility that mammalian Aph-1 genes are developmentally regulated. Recent studies indicated that mammalian APH-1 isoforms are differentially expressed in different organs-cell types in the adult animal (Hebert et al., 2004). The identification of a specific APH-1 isoform selectively expressed in the CNS during aging would have important therapeutic implica- 

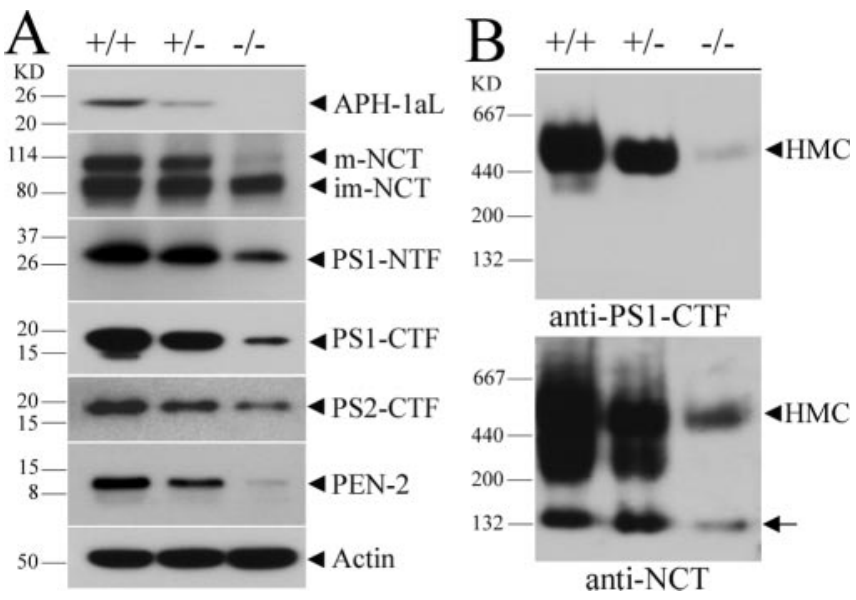

Figure 3. Levels of nicastrin, $\mathrm{PS} 1, \mathrm{PEN}-2$, and $\gamma$-secretase complex formation in $A p h-1 a^{-/-}$ cells. A, Protein extracts from immortalized $A p h-1 a^{+/+}, A p h-1 a^{+/-}$, and $A p h-1 a^{-/-}$fibroblasts were subjected to protein blot analysis using antisera specific to APH-1aL, NCT, PS1-NTF,

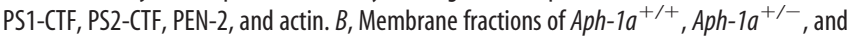
$A p h-1 a^{-1-}$ cells were prepared and subjected to blue native gel analysis using anti-NCT and anti-PS1 antibody. Both the levels of high-molecular-weight complex and APH-1/nicastrin subcomplex were reduced in Aph-1 $a^{-/-}$cells. HMC, High-molecular-weight $\gamma$-secretase complexes; arrow indicates APH- $1 /$ nicastrin subcomplexes.
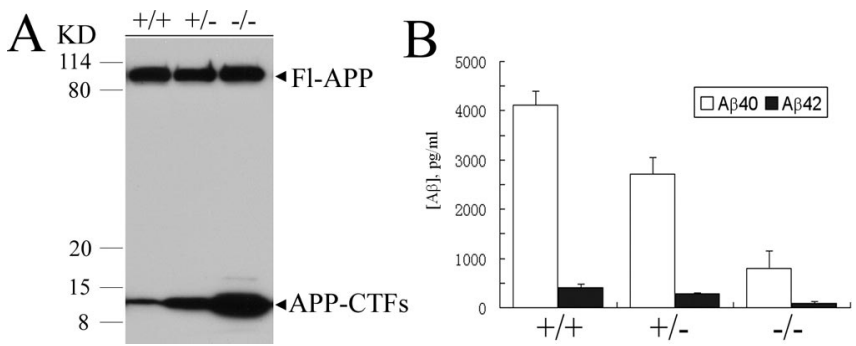

Figure 4. APP processing and secretion of A $\beta$ in $A p h-1 a^{-/-}$fibroblasts. $A, A p h-1 a^{+/+}$, $A p h-1 a^{+/-}$, and $A p h-1 a^{-1-}$ fibroblasts were infected with adenovirus expressing the human APP Swedish mutation for $36 \mathrm{hr}$, and extracts were subjected to protein blot analysis using CT15, an anti-APP C-terminal antibody. Fl-APP, Full-length APP; APP-CTFs, APP C-terminal fragments. The ratio of the APP-CTFs to full-length APP is significantly increased in Aph-1 $1 a^{-/-}$ cells. $B$, Conditioned media from Aph-1 $1 a^{+/+}, A p h-1 a^{+/-}$, and $A p h-1 a^{-/-}$fibroblasts were collected and analyzed by sandwich ELISA. Levels of secreted $A \beta 40$ and $A \beta 42$ were significantly reduced in $\mathrm{Aph}-1 a^{-/-}$cells.

tions for efforts to selectively alter $\gamma$-secretase activity to ameliorate $\beta$-amyloidosis in AD.

Although previous studies suggested that APH-1 and nicastrin form a precomplex that might facilitate assembly of the $\gamma$-secretase complex (Gu et al., 2003; LaVoie et al., 2003; Hu and Fortini, 2003; Takasugi et al., 2003), the molecular mechanism of precomplex assembly and whether APH-1 homologs are required to stabilize nicastrin to form the initial pre- $\gamma$-secretase complex remain elusive. Our findings that the deletion of Aph-1a significantly reduces the levels of mature nicastrin as well as APH$1 /$ nicastrin precomplex and high-molecular-weight complex coupled with the observation that APH-1 and nicastrin physically interact (Gu et al., 2003; Hu and Fortini, 2003; LaVoie et al., 2003; Morais et al., 2003) strongly support the view that APH-1 is necessary to stabilize nicastrin to form a stable precomplex. Together with the observation that the level of APH-1 is significantly reduced in nicastrin-deficient fibroblasts (Vetrivel et al., 2004), our data are consistent with a model that APH-1 and nicastrin are
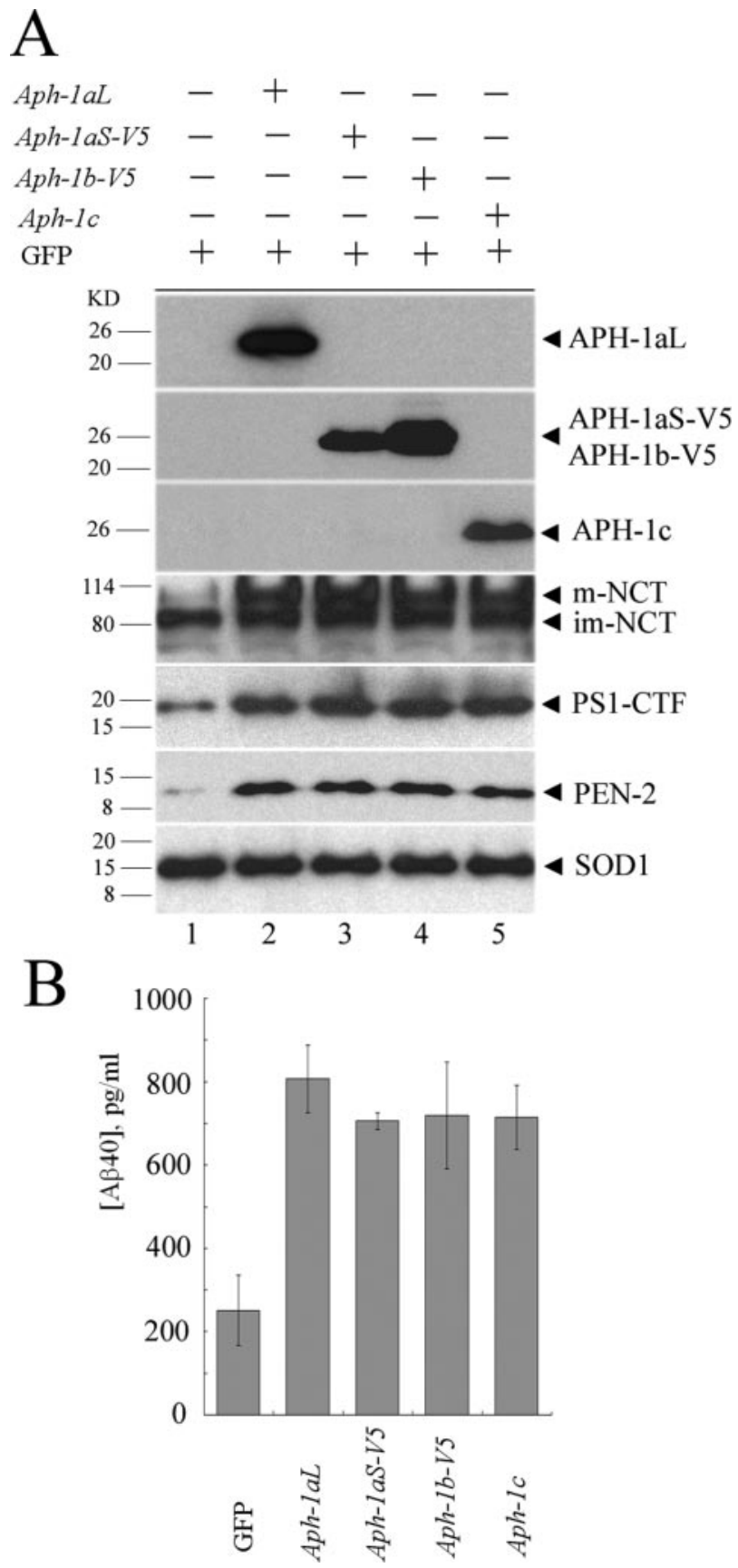

Figure 5. Reduced levels of the components of $\gamma$-secretase complex and enzymatic activity occurring in Aph-1 $1 a^{-/-}$cells can be complemented by expression of any APH- 1 isoforms. $A$, Immortalized Aph-1 $1 a^{-/-}$cells transiently transfected with expression plasmids encoding APH-1aL, APH-1aS-V5, APH-1b-V5, or APH-1c were infected with APP virus expressing human APP Swedish mutation $24 \mathrm{hr}$ after transfection. Cell extracts were prepared $48 \mathrm{hr}$ after transfection and subjected to protein blot analysis using anti-NCT, anti-PS1, and anti-PEN-2 antibodies. Cotransfection of expression vector encoding GFP (green fluorescent protein) was performed to estimate the efficiency of transfection (30-40\%). The expression of APH- 1 isoforms was analyzed using anti-APH-1aL antibody for APH-1aL, anti-V-5 antibody for APH-1aS and APH-1b, and anti-APH-1b/c for APH-1c. The blots were reprobed using antisera specific to SOD1. $B$, Conditioned media were collected $48 \mathrm{hr}$ after transfection ( $24 \mathrm{hr}$ after infection) and analyzed by quantitative sandwich ELISA. The levels of secreted $A \beta 40$ were significantly elevated in cultures of cells overexpressing APH-1aL, APH-1aS-V5, APH-1b-V5, or APH-1c.

required to regulate the stability of each other to form a stable precomplex that serves as a platform for assembling PS and PEN-2 to form a core $\gamma$-secretase complex.

Although recent studies showing that increased levels of active 
$\gamma$-secretase complex can be achieved by overexpression of APH-1, PEN-2, and nicastrin with PS (Kim et al., 2003; Kimberly et al., 2003; Takasugi et al., 2003) and that $\gamma$-secretase activity can be reconstituted in yeast (Edbauer et al., 2003) support the view that these four components constitute the minimal $\gamma$-secretase complex, it is not clear whether APH-1 isoforms serve to define a set of distinct $\gamma$-secretase complexes. Recent biochemical studies suggest that there may exist several distinct presenilin complexes (Gu et al., 2004) with presenilin dimer forming the core of the $\gamma$-secretase enzyme (Schroeter et al., 2003) and that six distinct $\gamma$-secretase complexes with different APH-1 isoforms can exist in cultured cells (Shirotani et al., 2004). Our demonstration that all murine APH-1 isoforms can complement the Aph-1a null phenotype in fibroblasts supports the idea that the four murine $\mathrm{APH}-1$ isoforms (APH-1aL, APH-1aS, APH-1b, and APH-1c) coupled with PS1 and PS2 define a set of eight distinct functional $\gamma$-secretase complexes. Consistent with this view is our finding that Aph-1b-deficient fibroblasts showed reduced levels of nicastrin, PS, and PEN-2, and, more importantly, the level of $\gamma$-secretase activity is directly proportional to the Aph-1 gene dosage (Li, Ma, Wen, Davenport, Price, and Wong, unpublished observations). Future studies directed at defining the role of APH-1 dependent $\gamma$-secretase complexes, particularly specific APH-1 isoform that may determine cleavage site selectivity on APP-CTF, would have important implications for development of specific anti-A $\beta$ therapy for AD.

In summary, the present investigation has established that $\mathrm{APH}-1 \mathrm{a}$ is the major APH-1 isoform required for $\gamma$-secretase activity during mouse embryonic development and that APH-1a is critical to stabilize nicastrin to form a pre- $\gamma$-secretase complex that facilitates the assembly of PS and PEN-2. It remains to be demonstrated whether a specific APH-1 isoform might be a valuable therapeutic target for $\mathrm{AD}$, a possibility that can be evaluated in mouse models of $\mathrm{A} \beta$ amyloidosis.

\section{References}

Cai H, Wang Y, McCarthy D, Wen H, Borchelt DR, Price DL, Wong PC (2001) BACE1 is the major $\beta$-secretase for generation of $\mathrm{A} \beta$ peptides by neurons. Nat Neurosci 4:233-234.

Conlon RA, Reaume AG, Rossant J (1995) Notch1 is required for the coordinate segmentation of somites. Development 121:1533-1545.

De Strooper B (2003) Aph-1, pen-2 and nicastrin with presenilin generate an active $\gamma$-secretase complex. Neuron 38:9-12.

Donoviel DB, Hadjantonakis AK, Ikeda M, Zheng H, Hyslop PS, Bernstein A (1999) Mice lacking both presenilin genes exhibit early embryonic patterning defects. Genes Dev 13:2801-2810.

Edbauer D, Winkler E, Regula JT, Pesold B, Steiner H, Haass C (2003) Reconstitution of $\gamma$-secretase activity. Nat Cell Biol 5:486-488.

Farmery MR, Tjernberg LO, Pursglove SE, Bergman A, Winblad B, Naslund J (2003) Partial purification and characterization of $\gamma$-secretase from postmortem human brain. J Biol Chem 278:24277-24284.

Fortna RR, Crystal AS, Morais VA, Pijak DS, Lee VM, Domas RW (2004) Membrane topology and nicastrin-enhanced endoproteolysis of APH-1, a component of $\gamma$-secretase complex. J Biol Chem 279:3685-3693.

Francis R, McGrath G, Zhang J, Ruddy DA, Sym M, Apfeld J, Nicoll M, Maxwell M, Hai B, Ellis MC, Parks AL, Xu W, Li J, Gurney M, Myers RL, Himes CS, Hiebsch R, Ruble C, Nye JS, Curtis D (2002) Aph-1 and pen-2 are required for notch pathway signaling, $\gamma$-secretase cleavage of $\beta A P P$, and presenilin protein accumulation. Dev Cell 3:85-97.

Goutte C, Tsunozaki M, Hale VA, Priess JR (2002) APH-1 is a multipass membrane protein essential for the Notch signaling pathway in Caenorhabditis elegans embryos. Proc Natl Acad Sci USA 99:775-779.

Gu Y, Chen F, Sanjo N, Kawarai T, Hasegawa H, Duthie M, Li W, Ruan X, Luthra A, Mount HT, Tandon A, Fraser PE, St. George-Hyslop P (2003) $\mathrm{APH}-1$ interacts with mature and immature forms of presenilins and nicastrin and may play a role in maturation of presenilin-nicastrin complexes. J Biol Chem 278:7374-7380.

Gu Y, Sanjo N, Chen F, Hasegawa H, Petit A, Ruan X, Li W, Shier C, Kawarai T, Schmitt-Ulms G, Westaway D, St. Geroge-Hyslop P, Fraser PE (2004) The presenilin proteins are components of multiple membrane-bound complexes that have different biological activities. J Biol Chem 279:31329-31336.

Hebert SS, Serneels L, Dejaegere T, Horre K, Dabrowski M, Baert V, Annaert W, Hartmann D, De Strooper B (2004) Coordinated and widespread expression of $\gamma$-secretase in vivo: evidence for size and molecular heterogeneity. Neurobiol Dis 17:260-272.

Herreman A, Hartmann D, Annaert W, Saftig P, Craessaerts K, Serneels L, Umans L, Schrijvers V, Checler F, Vanderstichele H, Baekelandt V, Dressel R, Cupers P, Huylebroeck D, Zwijsen A, Van Leuven FV, De Strooper B (1999) Presenilin 2 deficiency causes a mild pulmonary phenotype and no changes in amyloid precursor protein processing but enhances the embryonic lethal phenotype of presenilin 1 deficiency. Proc Natl Acad Sci USA 96:11872-11877.

Hu Y, Fortini ME (2003) Different cofactor activities in $\gamma$-secretase assembly: evidence for a nicastrin-Aph-1 subcomplex. J Cell Biol 161:685-690.

Huppert SS, Le A, Schroeter EH, Mumm JS, Saxena MT, Milner LA, Kopan R (2000) Embryonic lethality in mice homozygous for a processingdeficient allele of Notch1. Nature 405:966-970.

Kim SH, Ikeuchi T, Yu C, Sisodia SS (2003) Regulated hyperaccumulation of presenilin-1 and the " $\gamma$-secretase" complex. J Biol Chem 278:33992-34002.

Kimberly WT, LaVoie MJ, Ostaszewski BL, Ye W, Wolfe MS, Selkoe DJ (2003) $\gamma$-Secretase is a membrane protein complex comprised of presenilin, nicastrin, aph-1 and pen-2. Proc Natl Acad Sci USA 100:6382-6387.

LaVoie MJ, Fraering PC, Ostaszewski BL, Ye W, Kimberly WT, Wolfe MS, Selkoe DJ (2003) Assembly of the $\gamma$-secretase complex involves early formation of an intermediate subcomplex of Aph-1 and Nicastrin. J Biol Chem 278:37213-37222.

Lee SF, Shah S, Li H, Yu C, Han W, Yu G (2002) Mammalian APH-1 interacts with presenilin and nicastrin and is required for intramembrane proteolysis of amyloid- $\beta$ precursor protein and notch. J Biol Chem 277:45013-45019.

Lee SF, Shah S, Yu C, Wigley WC, Li H, Lim M, Pedersen K, Han W, Thomas P, Lundkvist J, Hao Y, Yu G (2004) A conserved GXXXG motif in APH-1 is critical for assembly and activity of the $\gamma$-secretase complex. J Biol Chem 279:4144-4152.

Li T, Ma G, Cai H, Price DL, Wong PC (2003) Nicastrin is required for assembly of presenilin $/ \gamma$-secretase complexes to mediate notch signaling and for processing and trafficking of $\beta$-amyloid precursor protein in mammals. J Neurosci 23:3272-3277.

Morais VA, Crystal AS, Pijak DS, Carlin D, Costa J, Lee VM, Domas RW (2003) The transmembrane domain region of nicastrin mediates direct interaction with APH- 1 and the $\gamma$-secretase complex. J Biol Chem 278:43284-43291.

Petersen RC (2003) Mild cognitive impairment clinical trials. Nat Rev Drug Discov 2:646-653.

Price DL, Sisodia SS (1998) Mutant genes in familial Alzheimer's disease and transgenic models. Annu Rev Neurosci 21:479-505.

Schagger H, von Jagow G (1991) Blue native electrophoresis for isolation of membrane protein complexes in enzymatically active form. Anal Biochem 199:223-231.

Schroeter EH, Ilagan MX, Brunkan AL, Hecimovic S, Li YM, Xu M, Lewis HD, Saxena MT, De Strooper B, Coonrod A, Tomita T, Iwatsubo T, Moore CL, Goate A, Wolfe MS, Shearman M, Kopan R (2003) A presenilin dimer at the core of the $\gamma$-secretase enzyme: insights from parallel analysis of Notch 1 and APP proteolysis. Proc Natl Acad Sci USA 100:13075-13080.

Selkoe D, Kopan R (2003) Notch and presenilin: regulated intramembrane proteolysis links development and degeneration. Annu Rev Neurosci 26:565-597.

Shirotani K, Edbauer D, Prokop S, Haass C, Steiner H (2004) Identification of distinct $\gamma$-secretase complexes with different APH-1 variants. J Biol Chem 279:41340-41345.

Sisodia SS, St. George-Hyslop PH (2002) $\gamma$-Secretase, Notch, A $\beta$ and Alzheimer's disease: where do the presenilins fit in? Nat Rev Neurosci 3: 281-290. 
Swiatek PJ, Lindsell CE, del Amo FF, Weinmaster G, Gridley T (1994) Notch1 is essential for postimplantation development in mice. Genes Dev 8:707-719.

Takasugi N, Tomita T, Hayashi I, Tsuruoka M, Niimura M, Takahashi Y, Thinakaran G, Iwatsubo T (2003) The role of presenilin cofactor in the $\gamma$-secretase complex. Nature 422:438-441.

Thinakaran G, Borchelt DR, Lee MK, Slunt HH, Spitzer L, Kim G, Ratovitsky T, Davenport F, Nordstedt C, Seeger M, Hardy J, Levey AI, Gandy SE, Jenkins NA, Copeland NG, Price DL, Sisodia SS (1996) Endoproteolysis of presenilin 1 and accumulation of processed derivatives in vivo. Neuron 17:181-190

Vetrivel KS, Cheng H, Sakurai T, Li T, Nukina N, Wong PC, Xu H, Thinaka- ran G (2004) Association of $\gamma$-secretase with lipid rafts in post-Golgi and endosome membranes. J Biol Chem 279:44945-44954.

Wolfe MS, Kopan R (2004) Intramembrane proteolysis: theme and variations. Science 305:1119-1123.

Wong PC, Cai H, Borchelt DR, Price DL (2002) Genetically engineered mouse models of neurodegenerative diseases. Nat Neurosci 5:633-639.

Yu G, Nishimura M, Arawaka S, Levitan D, Zhang L, Tandon A, Song YQ, Rogaeva E, Chen F, Kawarai T, Supala A, Levesque L, Yu H, Yang D, Holmes E, Miiman P, Liang Y, Zhang D, Xu D, Sato C, et al. (2000) Nicastrin modulates presenilin-mediated notch/glp-1 signal transduction and $\beta$ APP processing. Nature 407:48-54. 